\title{
Validation of the Korean Frailty Index in commu- nity-dwelling older adults in a nationwide Korean Frailty and Aging Cohort study
}

Hee-Won Jung ${ }^{1, *}$, Sunyoung Kim ${ }^{2, *}$, and Chang Won Won ${ }^{3}$

${ }^{1}$ Department of Internal Medicine, Seoul National University Hospital, Seoul; ${ }^{2}$ Department of Family Medicine, Kyung Hee University Medical Center, Seoul; ${ }^{3}$ Elderly Frailty Research Center, Department of Family Medicine, Kyung Hee University School of Medicine, Seoul, Korea

\section{Received: May 22, 2019 \\ Revised : June 30, 2019 \\ Accepted: July 23, 2019}

\section{Correspondence to}

Chang Won Won, Ph.D.

Elderly Frailty Research Center, Department of Family Medicine, Kyung Hee University School of Medicine, Kyungheedae-ro, Dongdaemun-gu, Seoul 02447, Korea

Tel: +82-2-958-8700

Fax: +82-2-958-8699

E-mail: chunwon62@naver.com https://orcid.org/0000-00026429-4461

*These authors contributed equally to this work.
Background/Aims: We aimed to assess validity of the Korean Frailty Index (KFI) and the modified KFI (mKFI) in nationwide Korean population as screening measures for frailty status in older adults.

Methods: Analysis was performed in the records of baseline assessments of 2,886 participants in the Korean Frailty Aging Cohort study from 2016 to 2017. The KFI included eight items on a history of hospitalization, self-reported health status, polypharmacy, weight loss, mood, incontinence, sensory problems, and timed up and go test. In mKFI, timed up and go test was replaced with a question whether a person can walk around a schoolyard. Cardiovascular Health Study (CHS) frailty scale was used as a gold standard.

Results: In study population (mean age, $76 ; 47.6 \%$ men), score of the KFI correlated with the CHS scale. The KFI correlated with common geriatric parameters including Activities of Daily Living, nutritional status, cognitive performance, and mood. As a construct validity, items of KFI correlated with CHS scale. As a criterion validity, sensitivity was $81.6 \%$, specificity was $67.0 \%$ to predict frailty by CHS scale with the score of 3 or higher in KFI. The KFI and mKFI correlated with each other $\left(R^{2}=0.88\right)$, and prediction ability for frailty by CHS scale was not significantly differed between KFI and mKFI.

Conclusions: The KFI and mKFI are valid instruments for frailty screening and might be useful as simple frailty screening tools to identify older adults who might benefit from comprehensive geriatric assessment and integrated, multidisciplinary geriatric care services.

Keywords: Frailty; Illness; Korea; Elderly; Incontinence

\section{INTRODUCTION}

Frailty is a common geriatric syndrome that occurs with systemic aging in older adults and is defined as a state of decreased physiological reserve, with increased vulnerability to possible stressors [1]. Based on extensive research to date, the spectrum of frailty shows large inter-individual heterogeneity among older adults; in addition, frailty status could predict geriatric health outcomes, including mortality, institutionalization, and functional decline, better than chronological age or traditional risk models [2-5]. Frailty status is associated with adverse outcomes in both community-dwelling older people $[6,7]$ and patients receiving medical therapy or undergoing surgical procedures $[8,9]$. Therefore, frailty status can offer guidance for individualized therapeutic 
decision making to provide tailored multidisciplinary geriatric care and prevent adverse geriatric outcomes in older adults $[7,10]$.

To meet this clinical need, there have been efforts to define frailty as a clinical entity and provide valid instruments for assessing frailty in older adults in both the research and clinical sectors [11]. One of the most extensively used definitions is the frailty phenotype operationalizing frailty as the presence of characteristic subcomponents of physical frailty, with the original suggestion of the Cardiovascular Health Study (CHS) frailty scale by Fried et al. [12]. However, the CHS frailty scale includes physical performance measures and questionnaires that are not easily performed in busy clinical settings, such as during screening. Another approach for measuring frailty is using a model of accumulation of deficit by counting the number of abnormal items in a comprehensive geriatric assessment (CGA) to produce a frailty index from o to $1[4,13]$. Although the frailty index calculated using this method can highly predict survival and can be used to calculate biological age in older adults [14,15], performing CGA in outpatient clinics is not feasible in Korea. Reflecting these difficulties, and to simplify frailty assessment in the clinical care of older adults, researchers have sought to provide easy-to-use instruments to screen frailty.

The Korean Frailty Index (KFI) was established to provide a simple scale for frailty screening in Korean older adults. The KFI was developed in 2010 by an expert panel of geriatricians including an internist, an epidemiologist, and four family physicians. The index was initially validated using the CHS frailty scale as a gold standard in 240 older people from three community senior welfare centers [16]. The KFI covers various domains of geriatric assessment, with eight items including a history of hospitalization, subjective sense of healthiness, polypharmacy including Korean herbal medicines, subjective weight loss in the previous month, questions on depression and incontinence, the timed up and go (TUGT) test, and a question regarding visual and hearing impairments. Although the KFI initially showed a correlation with the CHS frailty scale, with a kappa of 0.50 , the initial validation study was performed in a rather homogeneous population who were able to visit welfare centers in a single urban area; thus, panelists in the original study called for further improvements in the
KFI and larger validation studies in a nationwide Korean population [6].

In this study, we aimed to validate the KFI in the Korean Frailty Aging Cohort Study (KFACS), a nationwide multicenter-based cohort, to study the prevalence and natural course of frailty in Korean community-dwelling older adults [17]. We also aimed to provide optimal cutoff values of the KFI to screen prefrailty and frailty, using previously validated measures of frailty, including the CHS frailty scale and the frailty index.

\section{METHODS}

\section{Study population and protocol}

This was a cross-sectional study, and participants consisted of older adults aged 70 to 84 years who participated in the KFACS. The participants were recruited from community-dwelling residents in urban and rural regions nationwide, based on age- and gender-specific strata. Candidates were recruited by personal contact from public health centers, senior centers, residence (apartment or house) in the community. Baseline evaluations were performed at 10 study sites that included eight. The KFACS is a nationwide cohort study that began in 2016 for the purpose of identifying and preventing factors that may contribute to frailty in community-dwelling older adults. The KFACS recruited 3,014 older adults for a baseline survey conducted from 2016 to 2017 at 10 centers throughout the country. Anthropometries were measured by an expert inspector trained in standardized measurement methods to minimize possible interrater variabilities. An in-person interview and health examinations were performed.

Community residents with no plans to move in the following 2 years and with no difficulties in conversing were eligible to participate in this study [17]. Uncontrolled hypertension (> 180/100 $\mathrm{mmHg}$ ), cerebrovascular accident or myocardial infarction within the past 6 months, and patients with active malignancy currently under therapeutic treatment were excluded. Of a total 3,014 participants recruited during the first 2 years, 2,886 participants who completed the survey were included in the final analysis. 


\section{Korean Frailty Index}

The KFI was developed in 2010 by a consensus panel of the Korean Geriatrics Society, and validated using the CHS frailty scale among 240 community-dwelling older adults at three senior welfare centers in Seoul [16]. The KFI includes eight items (Supplementary Table 1), querying a history of hospitalization in the previous 1 year, self-reported health status, polypharmacy, weight loss, depressive mood, incontinence, visual or auditory problems, and physical performance measured using the TUGT. For hospitalization, a history of 1 or more admissions to any kind of hospital receives 1 point. For self-reported health status, answering "poor" to the question "How do you think your current health status is?" receives 1 point. For weight loss, answering "yes" to the question "Have you experienced weight loss in the past year to the extent that your clothes fit loosely?" receives 1 point. For polypharmacy, taking four or more medications regularly receives 1 point. For depressive mood, answering "sometimes," "mostly," or "always" to the question "Have you experienced sadness or depressed mood during the previous 1 month?" receives 1 point. For incontinence, answering "sometimes," "mostly," or "always" to the question "Have you experienced incontinence of urine or feces in the previous 1 month?" receives 1 point. For visual or auditory problems, answering "yes" to the question "Do you have any problems with decreased visual acuity or difficulties with hearing in daily life?" receives 1 point. If the TUGT takes more than 10 seconds, the participant receives 1 point for decreased physical performance. In the original validation study, cut points for prefrailty and frailty were 3 points or higher and 5 points or higher, respectively.

In the present study, we used the original version of the KFI to assess correlations of the measures with the CHS frailty scale and common geriatric conditions. In addition, to facilitate the use of the KFI in a busy clinical practice and community-based research, we established and validated a modified KFI (mKFI), replacing the TUGT with a question asking whether the person is able to walk around a standard-sized schoolyard (400 $\mathrm{m}$ ) without difficulty. This replacement was purposed to minimize requirement for physical examination during assessments for frailty, and to make possible for phonecall based assessments or interview based assessments from caretakers.

\section{CHS frailty scale}

For the definition of frailty, we used the Fried phenotype, which comprises five components: unintended weight loss, poor grip strength, exhaustion, reduced walking speed, and low physical activity level [12]. For unintended weight loss, 1 point was given for unintended weight loss of $4.5 \mathrm{~kg}$ or more in the previous year. Grip strength was measured using a hand dynamometer (Takei TKK 5401, Takei Scientific Instruments, Tokyo, Japan). In the first round of measurement, the grip strength of each hand was measured once. A second round of measurement was performed after 3 minutes, in which the grip strength of each hand was measured again in an alternate manner. The highest value out of four measurements was used in the analysis. One point was given for a grip strength less than $26 \mathrm{~kg}$ in men or less than $18 \mathrm{~kg}$ in women [18]. To quantify exhaustion, 1 point was given when the participant responded affirmatively to either of the following statements from the Center for Epidemiological Studies-Depression scale for 3 or more days in a week: "I felt that everything I did was an effort" or "I could not get going" [19]. Fried et al. [12] defined "CHS slowness" as the slowest $20 \%$ of a cohort (by sex and height), but for the sake of convenience, we set the cutoff for slow gait speed to be $1 \mathrm{~m} / \mathrm{sec}$, with a walking speed $4 \mathrm{~m} / \mathrm{sec}$ as the usual gait speed. For low physical activity level, 1 point was given for physical activity resulting in energy expenditure below $494.6 \mathrm{kcal}$ per week for men and below $283.5 \mathrm{kcal}$ per week for women, according to the International Physical Activity Questionnaire. These values correspond to the lowest $20 \%$ of the sex-specific total energy consumed according to a general population-based survey of older adults [20]. Participants with a total score of 3 or more were classified as frail; those with a total score of 1 to 2 were classified as prefrail, and those with no points for any of the criteria were classified as robust.

\section{Other physical performance and muscle mass measurement}

The short physical performance battery (SPPB), in which balance, walking, and the ability to rise from a sitting position in a chair, were administered based on the existing recommendations [21]. Each item of the SPPB is scored based on a o to 4-point scale, with a total score ranging from $o$ to 12 points. To assess the ability to rise 
from a chair, the time taken to complete five repetitions of sitting in a chair and getting up from it was measured.

For the assessment of appendicular skeletal muscle mass (ASM), dual energy X-ray absorptiometry (Hologic DXA, Hologic Inc., Bedford, MA, USA; and Lunar, GE Healthcare, Madison, WI, USA) was used. The skeletal muscle mass index was calculated as ASM divided by height squared $\left(\mathrm{m}^{2}\right)$.

\section{Covariates}

Information on age, marital status, education level, drinking status, smoking status, number of medications, comorbidities, and scores for the Korean Instrumental Activities of Daily Living [22], Korean Mini-Mental State Examination (MMSE) [23], EuroQol-5 dimensions [24], Geriatric Depression Scale Short Form [25], and Minimal Nutritional Assessment questionnaires [26] was collected during face-to-face interviews. Alcohol consumption was defined as three or more alcoholic drinks per week, and smoking was defined as a lifetime consumption of 100 or more cigarettes.

\section{Ethical approval}

Our research plan was approved by the Institutional Review Board of Kyung Hee University, and written informed consent was obtained from each participant prior to commencement of the study (Approval no.: KMC IRB 2019-04-069).

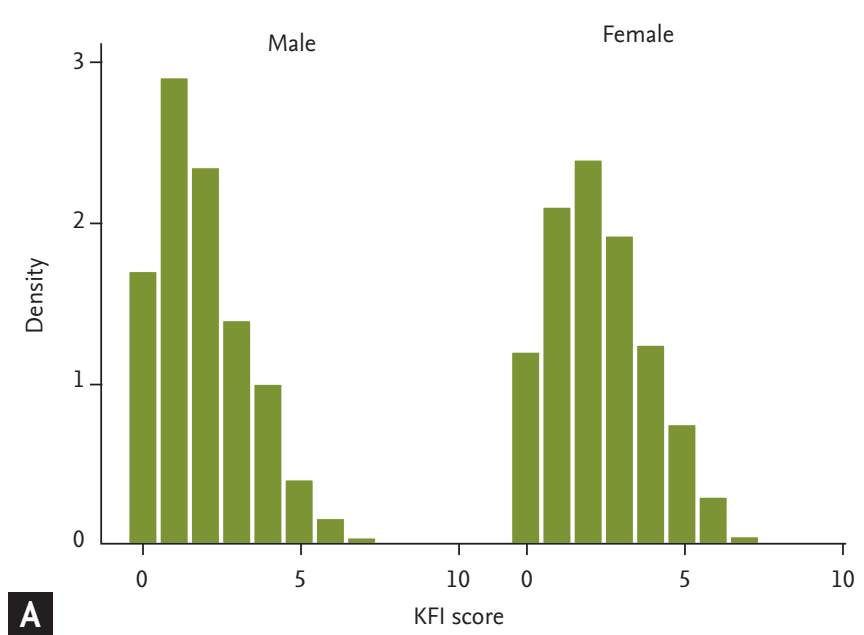

\section{Statistical methods}

Normally distributed continuous variables were expressed as mean \pm standard deviation, and categorical variables were expressed as number and percentage. For continuous variables, $t$ tests were performed and we used chi-square tests for categorical variables. We used Spearman's rho to examine the relationships between each component of the KFI and frailty according to the CHS frailty scale. Receiver operating characteristic (ROC) analyses were also performed to identify the cutoff values for KFI scores. IBM SPSS version 23.0 (IBM Corp., Chicago, IL, USA) and Stata version 15.0 (StataCorp., College Station, TX, USA) were used for all statistical analyses, and statistical significance was set at a two-sided $p$ value less than 0.05 .

\section{RESULTS}

\section{General characteristics and distributions of the KFI}

The mean age of included participants was 76 years, and 1,374 (47.6\%) were men. Among the 2,886 participants, $1,311(45.4 \%)$ were classified as robust, 1,341 (46.5\%) as prefrail, and $234(8.1 \%)$ as frail, based on the CHS frailty scale. The mean of the KFI was higher in women (mean, $2.4 \pm 1.6$ ) than in men (mean, $1.9 \pm 1.5 ; p<0.001$ ) (Fig. 1). Age, alcohol, smoking, education, presence of a spouse, number of comorbidities, physical activity, skeletal muscle mass index, body mass index, and physical activity, were significantly different by sex $(p<0.001)$ (Table

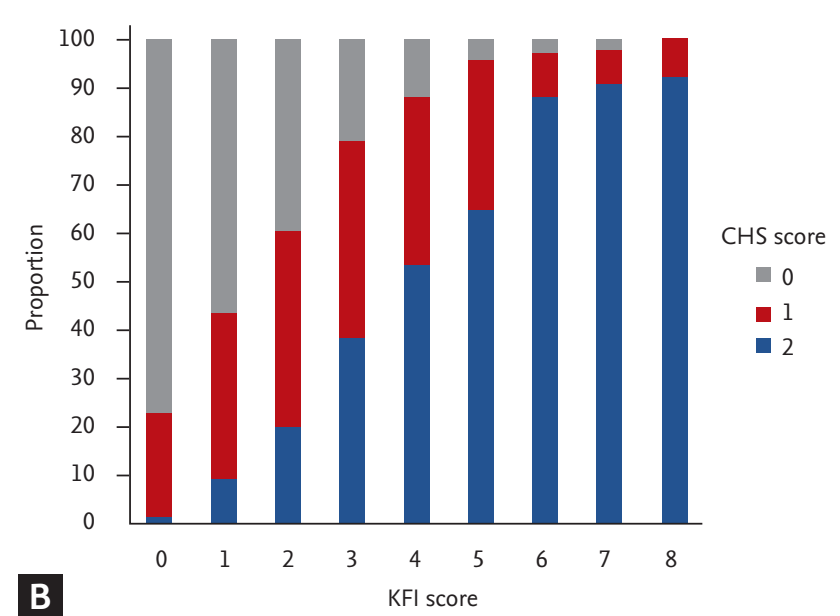

Figure 1. (A) Distribution of the Korean Frailty Index (KFI) by sex, and (B) correlation between the KFI and Cardiovascular Health Study (CHS) frailty scale scores. 
Table 1. General characteristics of participants $(n=2,886)$

\begin{tabular}{|c|c|c|c|c|}
\hline Characteristic & Total $(\mathrm{n}=2,886)$ & $\operatorname{Men}(n=1,374)$ & Women $(\mathrm{n}=1,512)$ & $p$ value \\
\hline Age, yr & $76.0 \pm 3.9$ & $76.3 \pm 3.9$ & $75.7 \pm 3.9$ & $<0.001$ \\
\hline Education, > 6 yr & $2,287(79 \cdot 5)$ & $1,247(91.1)$ & $1,040(68.8)$ & $<0.001$ \\
\hline Alcohol consumption & $523(18.1)$ & $455(33.1)$ & $68(4 \cdot 5)$ & $<0.001$ \\
\hline Smoking & $1,180(38.4)$ & $1,068(77.7)$ & $40(2.6)$ & $<0.001$ \\
\hline Presence of spouse & $1,940(67.2)$ & $1,229(89.4)$ & $711(47 \cdot 0)$ & $<0.001$ \\
\hline Physical activity, kcal/wk & $3,361.1 \pm 4,100.8$ & $4,291.4 \pm 4,994 \cdot 1$ & $2,515.2 \pm 2,818.2$ & $<0.001$ \\
\hline No. of comorbidities & $2.4 \pm 1.7$ & $2.2 \pm 1.6$ & $2.6 \pm 1.7$ & $<0.001$ \\
\hline Activities of daily living & $7.1 \pm 0.4$ & $7.1 \pm 0.4$ & $7.1 \pm 0.4$ & 0.002 \\
\hline Nutritional status, MNA score & $12.9 \pm 1.5$ & $12.9 \pm 1.5$ & $12.8 \pm 1.5$ & 0.328 \\
\hline Cognitive function, MMSE score & $25 \cdot 6 \pm 3 \cdot 3$ & $26.2 \pm 2.9$ & $25.1 \pm 3 \cdot 5$ & $<0.001$ \\
\hline Depressive status, GDS score & $3.2 \pm 3 \cdot 7$ & $2.4 \pm 3.2$ & $3.8 \pm 3.9$ & $<0.001$ \\
\hline Quality of life, EQ-5D score & $0.9 \pm 0.1$ & $0.9 \pm 0.1$ & $0.9 \pm 0.1$ & $<0.001$ \\
\hline Body mass index, $\mathrm{kg} / \mathrm{m}^{2}$ & $24.5 \pm 3.0$ & $24.0 \pm 2.9$ & $24.9 \pm 3.1$ & $<0.001$ \\
\hline Gait speed, $\mathrm{m} / \mathrm{sec}$ & $1.2 \pm 0.3$ & $1.2 \pm 0.3$ & $1.1 \pm 0.2$ & $<0.001$ \\
\hline Grip strength, kg & $26.3 \pm 7.6$ & $32.2 \pm 6.0$ & $20.9 \pm 4.2$ & $<0.001$ \\
\hline Timed up and go test, sec & $10.5 \pm 2.8$ & $10.2 \pm 2.7$ & $10.7 \pm 2.9$ & $<0.001$ \\
\hline Five-repetition sit-to-stand test, sec & $11.4 \pm 4.0$ & $10.7 \pm 3.4$ & $12.0 \pm 4.3$ & $<0.001$ \\
\hline CHS frailty scale & $0.9 \pm 1.0$ & $0.7 \pm 1.0$ & $1.0 \pm 1.0$ & $<0.001$ \\
\hline KFI score & $2.2 \pm 1.6$ & $1.9 \pm 1.5$ & $2.4 \pm 1.6$ & $<0.001$ \\
\hline mKFI score & $2.0 \pm 1.6$ & $1.7 \pm 1.5$ & $2.3 \pm 1.6$ & $<0.001$ \\
\hline
\end{tabular}

Values are presented as mean \pm SD or number (\%). Alcohol consumption defined as three or more alcoholic drinks a week, and smoking defined as lifetime consumption of 100 or more cigarettes. Activities of daily living (range 7 to 21, increasingly worse), geriatric depression scale (range o to 15 , increasingly worse).

MNA, Mini Nutritional Assessment; MMSE, Mini-Mental State Examination; GDS, Geriatric Depression Scale; EQ-5D, EuroQol-5 dimensions; CHS, Cardiovascular Health Study; KFI, Korean Frailty Index; mKFI, modified Korean Frailty Index.

1). The frailty scores of KFI, mKFI and CHS frailty scale were higher in the rural area than in the urban area, and statistical significances between dwelling status were highest in CHS frailty scale and lowest in KFI (Supplementary Table 1).

\section{Content validity}

The KFI score (o to 8) was positively associated with the CHS frailty score (o to 5) (rho $=0.51 ; p<0.001)$ (Fig. 2B). KFI score correlated not only with physical function such as short physical performance battery, grip strength, gait speed, and five-repetition sit-to-stand test (rho ranging, -0.42 to $0.50 ; p<0.001)$ but also with Activities of Daily Living (ADL; rho $=0.29, p<0.001$ ), nutritional status (rho $=-0.25, p<0.001)$, MMSE (rho $=-0.26, p<0.001$ ), and $\operatorname{mood}($ rho $=0.52, p<0.001)$ (Table 2). Correlations be- tween the KFI score and common geriatric parameters are shown in Fig. 2.

\section{Construct validity}

The internal consistency and kappa coefficients between frailty based on the KFI (each domain and total score) and frailty according to the CHS frailty scale are presented in Table 3. The kappa coefficients of the eight items on the KFI were all statistically significant with respect to frailty according to the CHS frailty scale $(p<$ 0.001); kappa coefficients of the KFI items ranged from 0.053 to 0.223 . Among these eight items, the most sensitive to the corresponding item of the KFI was TUGT (sensitivity 94.9\%), and the least sensitive was history of hospital admission (sensitivity 18.8\%). 

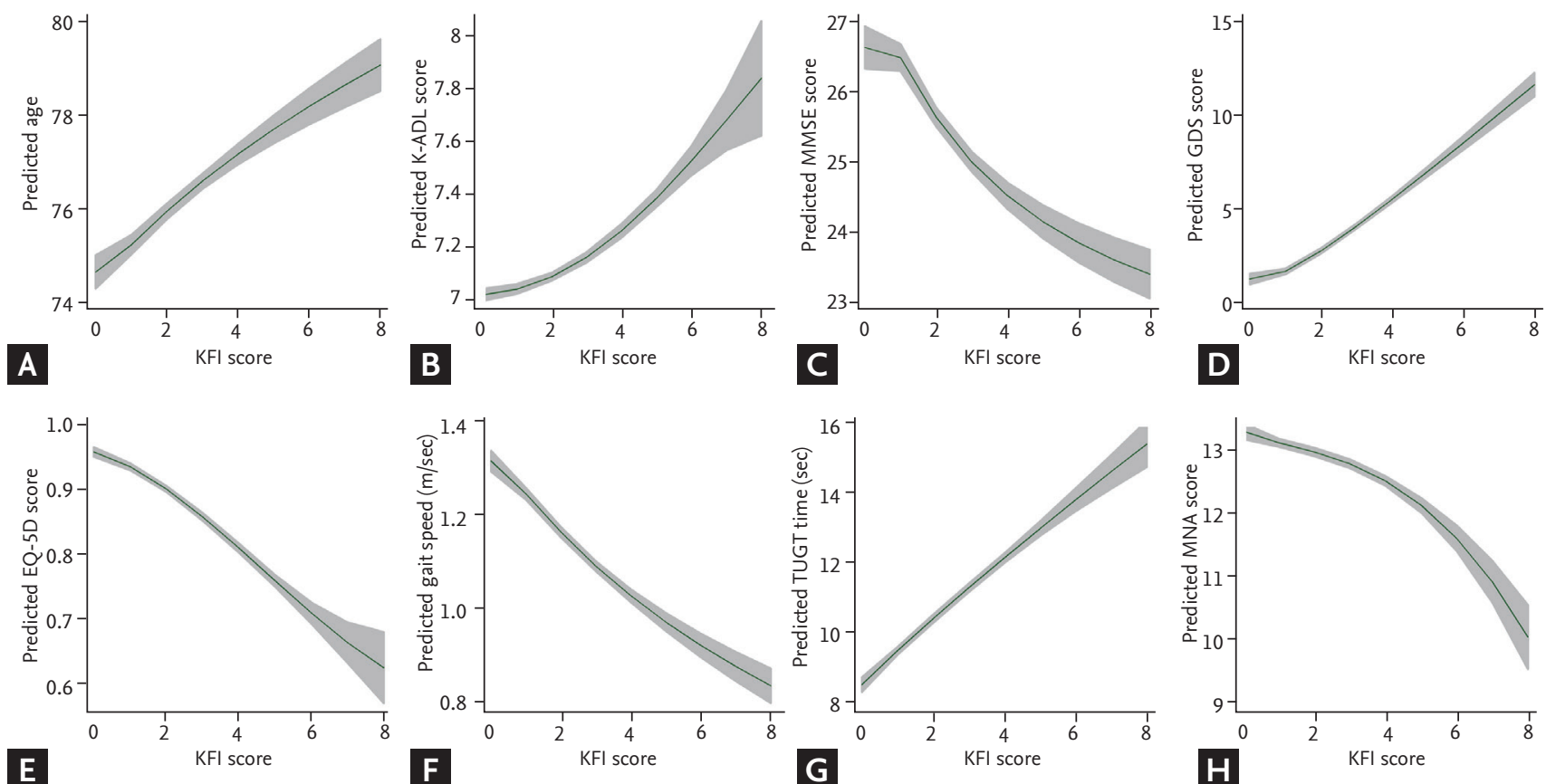

Figure 2. (A-H) Distributions of modified Korean Frailty Index (mKFI) and measurements. K-ADL, Korean Activities of Daily Living; MMSE, Mini-Mental State Examination; GDS, Geriatric Depression Scale; EQ-5D, EuroQol-5 dimensions; TUGT, timed up and go test; MNA, Mini Nutritional Assessment.

Table 2. Validation between the KFI and other measurements

\begin{tabular}{|c|c|c|c|c|}
\hline \multirow{2}{*}{ Variable } & \multicolumn{2}{|c|}{ KFI score } & \multicolumn{2}{|c|}{ Age- and sex-adjusted KFI score } \\
\hline & rho & $p$ value & rho & $p$ value \\
\hline CHS frailty score & 0.512 & $<0.001$ & 0.454 & $<0.001$ \\
\hline Grip strength & -0.292 & $<0.001$ & -0.210 & $<0.001$ \\
\hline Gait speed & -0.423 & $<0.001$ & -0.345 & $<0.001$ \\
\hline Timed up and go test & 0.497 & $<0.001$ & 0.466 & $<0.001$ \\
\hline Five-repetition sit-to-stand test & 0.371 & $<0.001$ & 0.316 & $<0.001$ \\
\hline SPPB score & -0.409 & $<0.001$ & -0.345 & $<0.001$ \\
\hline MNA score & -0.252 & $<0.001$ & -0.241 & $<0.001$ \\
\hline MMSE score & -0.262 & $<0.001$ & -0.185 & $<0.001$ \\
\hline GDS score & 0.522 & $<0.001$ & 0.489 & $<0.001$ \\
\hline EQ-5D score & -0.493 & $<0.001$ & -0.447 & $<0.001$ \\
\hline K-ADL score & 0.292 & $<0.001$ & 0.275 & $<0.001$ \\
\hline Skeletal muscle index & -0.107 & $<0.001$ & -0.021 & 0.258 \\
\hline
\end{tabular}

Correlations were analyzed using the Spearman test.

KFI, Korean Frailty Index; CHS, Cardiovascular Health Study; SPPB, Short Physical Performance Battery; MNA, Mini Nutritional Assessment; MMSE, Mini-Mental State Examination; GDS, Geriatric Depression Scale; EQ-5D, EuroQol-5 dimensions; K-ADL, Korean activities of daily living. 
Table 3. Agreement of KFI items with frailty using the CHS frailty scale

\begin{tabular}{|c|c|c|c|c|c|c|c|c|c|}
\hline \multirow[b]{2}{*}{ KFI items } & \multicolumn{2}{|c|}{ CHS frailty } & \multirow[b]{2}{*}{$\begin{array}{c}\text { Sensitivity, } \\
\%\end{array}$} & \multirow[b]{2}{*}{$\begin{array}{c}\text { Specificity, } \\
\%\end{array}$} & \multirow[b]{2}{*}{$\begin{array}{c}\text { Accuracy, } \\
\%\end{array}$} & \multirow[b]{2}{*}{$\begin{array}{l}\text { PPV, } \\
\%\end{array}$} & \multirow[b]{2}{*}{$\begin{array}{l}\text { NPV, } \\
\%\end{array}$} & \multirow[b]{2}{*}{ Kappa } & \multirow[b]{2}{*}{$p$ value } \\
\hline & $\begin{array}{l}\text { No frailty } \\
(\mathrm{n}=2,652)\end{array}$ & $\begin{array}{l}\text { Frailty } \\
(\mathrm{n}=234)\end{array}$ & & & & & & & \\
\hline Hospital admission & & & 18.8 & 87.9 & 82.3 & 12.0 & 92.5 & 0.053 & 0.009 \\
\hline No & $2,330(87 \cdot 9)$ & $190(81.2)$ & & & & & & & \\
\hline Yes & $322(12.1)$ & $44(18.8)$ & & & & & & & \\
\hline Self-assessment of health status & & & 68.8 & $73 \cdot 4$ & 73.0 & 18.6 & 96.4 & 0.189 & $<0.001$ \\
\hline No & $1,946(73 \cdot 4)$ & $73(31.2)$ & & & & & & & \\
\hline Yes & $706(26.6)$ & $161(68.8)$ & & & & & & & \\
\hline Polypharmacy & & & 65.8 & 56.2 & $57 \cdot 0$ & 11.7 & $94 \cdot 9$ & 0.071 & $<0.001$ \\
\hline No & $1,490(56.2)$ & $80(34.2)$ & & & & & & & \\
\hline Yes & $1,162(43.8)$ & $154(65.8)$ & & & & & & & \\
\hline Weight loss & & & 26.5 & $93 \cdot 4$ & 88.0 & 26.3 & $93 \cdot 5$ & 0.199 & $<0.001$ \\
\hline No & $2,478(93.4)$ & $172(73.5)$ & & & & & & & \\
\hline Yes & $174(6.6)$ & $62(26.5)$ & & & & & & & \\
\hline Depressed mood & & & 58.6 & 67.5 & 66.7 & $13 \cdot 7$ & $94 \cdot 9$ & 0.104 & $<0.001$ \\
\hline No & $1,789(67.5)$ & $97(41.5)$ & & & & & & & \\
\hline Yes & $863(32.5)$ & $137(58.5)$ & & & & & & & \\
\hline Incontinence & & & 28.3 & $85 \cdot 3$ & 80.7 & $14 \cdot 5$ & 93.1 & 0.095 & $<0.001$ \\
\hline No & $2,263(85 \cdot 3)$ & $167(71.7)$ & & & & & & & \\
\hline Yes & $389(14.7)$ & $66(28.3)$ & & & & & & & \\
\hline Timed up and go test & & & 94.9 & 56.0 & 59.1 & 16.0 & 99.2 & 0.156 & $<0.001$ \\
\hline No & $1,484(56.0)$ & $12(5 \cdot 1)$ & & & & & & & \\
\hline Yes & $1,168(44.0)$ & $222(94.9)$ & & & & & & & \\
\hline Visual or auditory problems & & & $39 \cdot 3$ & 80.7 & $77 \cdot 4$ & $15 \cdot 3$ & 93.8 & 0.117 & $<0.001$ \\
\hline No & $2,141(80.7)$ & $142(60.7)$ & & & & & & & \\
\hline Yes & $511(19 \cdot 3)$ & $92(39 \cdot 3)$ & & & & & & & \\
\hline Walking $400 \mathrm{~m} / \mathrm{schoolyard}$ & & & 77.8 & 73.2 & 73.6 & 20.4 & $97 \cdot 4$ & 0.223 & $<0.001$ \\
\hline No & $1,941(73.2)$ & $52(22.2)$ & & & & & & & \\
\hline Yes & $711(26.8)$ & $182(77.8)$ & & & & & & & \\
\hline
\end{tabular}

Values are presented as number (\%).

KFI, Korean Frailty Index; CHS, Cardiovascular Health Study; PPV, positive predictive value; NPV, negative predictive value.

\section{Criterion validity}

ROC analysis performed to confirm the criterion-related validity of the KFI for frailty according to the CHS frailty scale showed an area under the ROC curve (AUC) of 0.82 (95\% confidence interval [CI], 0.79 to 0.84 ); according to the maximum Youden index, the KFI score for predicting frailty was 3 or higher (sensitivity, 81.6\%; specificity, 67.0\%). For the KFI in terms of predicting vulnerability (prefrailty + frailty) according to the CHS frailty scale, the AUC of ROC was 0.74 (95\% CI, 0.72 to
0.76); the KFI score to predict vulnerability according to the maximum Youden index was 2 or higher (sensitivity, 76.1\%; specificity, 62.8\%). When these cut points were used, the prevalence of frailty and prefrailty in the study population according to the KFI were $8.1 \%$ and $46.5 \%$, respectively.

\section{Comparisons of the mKFI and KFI}

We assessed whether the mKFI can be also used to screen frailty, similar to the KFI. In linear regression 
Table 4. Sensitivity and specificity for the KFI and modified KFI cutoffs in predicting frailty based on the CHS frailty scale (ROC analysis)

\begin{tabular}{|c|c|c|c|c|}
\hline Cutoff & AUC & Sensitivity, \% & Specificity, \% & Youden index, \% \\
\hline KFI & $0.82(0.79-0.84)$ & & & \\
\hline$\geq 1$ & & 99.6 & $15 \cdot 6$ & 15.2 \\
\hline$\geq 2$ & & $94 \cdot 4$ & $42 \cdot 3$ & 36.7 \\
\hline$\geq 3$ & & 81.6 & 67.0 & 48.6 \\
\hline$\geq 4$ & & 61.1 & 83.4 & 44.6 \\
\hline$\geq 5$ & & 37.6 & 93.7 & 31.3 \\
\hline$\geq 6$ & & 20.9 & 98.6 & $19 \cdot 5$ \\
\hline$\geq 7$ & & 4.7 & 99.7 & $4 \cdot 4$ \\
\hline$\geq 8$ & & 0.9 & 100 & 0.8 \\
\hline Modified KFI & $0.81(0.78-0.84)$ & & & \\
\hline$\geq 1$ & & 98.7 & 20.3 & 19.0 \\
\hline$\geq 2$ & & 89.7 & 48.6 & 38.3 \\
\hline$\geq 3$ & & 76.9 & 70.6 & $47 \cdot 5$ \\
\hline$\geq 4$ & & $57 \cdot 7$ & 85.6 & $43 \cdot 3$ \\
\hline$\geq 5$ & & $35 \cdot 5$ & $94 \cdot 3$ & 29.8 \\
\hline$\geq 6$ & & 19.7 & 98.5 & 18.2 \\
\hline$\geq 7$ & & 4.7 & 99.7 & $4 \cdot 4$ \\
\hline$\geq 8$ & & 0.9 & 100 & 0.9 \\
\hline
\end{tabular}

KFI, Korean Frailty Index; CHS, Cardiovascular Health Study; ROC, receiver operating characteristic; AUC, area under the ROC curve.

analysis, the mKFI and KFI correlated with each other (B $\left.=0.92, R^{2}=0.88, p<0.001\right)$. According to the mKFI, AUCs for vulnerability and frailty by the CHS frailty scale were 0.73 (95\% CI, 0.62 to $0.75 ; p=0.158$ when compared with the KFI) and 0.81 ( $95 \%$ CI, 0.78 to $0.83 ; p=0.05$ when compared with the KFI), respectively. When similar cutoffs as the KFI were applied for the mKFI, sensitivities/specificities were $76.9 \% / 70.6 \%$ for frailty and $70.9 \% / 65.1 \%$ for vulnerability, both according to the CHS scale (Table 4).

\section{DISCUSSION}

In this study, we found that both the original KFI and the mKFI were valid as frailty assessment instruments among community-dwelling older adults in terms of content, construct, and criterion validity, when compared with the extensively studied CHS frailty scale. In both the original KFI and mKFI, in which scores range from o to 8, individuals with a score of 2 or higher might be considered prefrail and those scoring 3 or higher are considered frail. Although the KFI and MKFI were designed with sub-items reflecting domains of CGA, we recognize that these measures might be used to screen physical frailty in older adults.

Although there is accumulating research evidence on the clinical importance of frailty in terms of adverse health outcomes in the Korean population, appropriate and easy-to-use methods to screen frailty that have been validated in a nationwide manner are lacking in Korea. In relevant research, a frailty index using geriatric assessment data has been widely used to predict clinical outcomes among older adults, with intrinsically high outcome predictability owing to its large number of items (usually > 30 items) [3,10]. Recognizing that conducting geriatric assessment in general populations of older people is not feasible, Jung et al. [27] suggested use of the Korean version of the Fatigue, Resistance, Ambulation, Illness, and Loss of weight (FRAIL) scale, which has been validated in diverse older Korean populations, as a screening measure for frailty [28]. However, four of five items on the FRAIL scale are allocated to physical 
frailty, limiting its ability to capture deficits of functional capacity in daily living. Moreover, the items querying illness, to quantify comorbidity, was based on older Western populations, and the positive rate of this item is less than $5 \%$ in the Korean population [27]. Therefore, the FRAIL scale might not be an optimal measure to screen frailty in older Korean adults who might benefit from an in-depth CGA and downstream care planning. In contrast, the KFI was designed as an abbreviated CGA entailing objective (hospital admission, polypharmacy) and subjective health status (weight loss, mood, continence, and physical performance) $[16,29]$. Therefore, compared with the FRAIL scale, the KFI might be more suitable for screening older prefrail/frail people who may require a multidisciplinary geriatric approach for unrecognized functional deficits in the real world. Indeed, in our analysis, the KFI correlated in a dose-response manner with measures of CGA such as ADL, IADL, gait speed, cognition, mood, nutrition, and quality of life.

To enable easier screening of frailty and do away with the need for physical measurement in participant/patients, we replaced the TUGT with a question on mobility in the mKFI, that is, whether the person can walk around an average-sized schoolyard without any difficulty. Although the mobility question did not completely agree with the TUGT in the study population, the screening performance of the mKFI for vulnerability was not statistically different from that of the KFI. Therefore, in clinical research among older adults with continuous follow-up for frailty and functional status [30], the mKFI might be easy to use via telephone calls or smartphone-based self-administered questionnaires.

Using ROC analysis, we found that the suggested cutoff values of prefrailty and frailty for KFI and mKFI scores could be 2 and 3, respectively. However, in an original study by Hwang et al. [16], the suggested cutoff values for prefrailty and frailty were 3 or higher (sensitivity $74.2 \%$, specificity $63.9 \%$ ) and 5 or higher (sensitivity $82.1 \%$, specificity $86.8 \%$ ), respectively. This discrepancy might be attributable to differences in study populations between these two studies. In addition, because there was no population-specific consensus for slow gait speed and low grip strength in 2010, Hwang et al. [16] had to classify the grip strength and gait speed of the lowest quintile in the study population as slowness and weakness on the CHS frailty scale; this is in contrast to the present study, which used the Asian Working Guideline on Sarcopenia consensus cutoff values. Regarding differences in study design and generalizability to the Korean population, revised cutoff values for the KFI and mKFI might be better in screening frailty among community-dwelling Korean older adults.

Although this study is the first to validate the KFI and $\mathrm{mKFI}$ in a nationwide population of Korean older adults, some limitations exist. As the questionnaire was administered to participants by skilled research nurses who are familiar with collecting information from older people in geriatric assessments of the KFACS, the feasibility of the KFI and mKFI in busy clinical practices cannot be assured from the findings of the present study; this question should be evaluated in a future real-world-based clinical study. In addition, we could not assess the outcome relevance of the KFI and mKFI because this cross-sectional study used records of baseline assessments in the KFACS. Further analyses on geriatric outcomes using upcoming KFACS data may provide further information on the outcome validity of the KFI and $\mathrm{mKFI}$.

In conclusion, we found that the KFI and mKFI are valid instruments for frailty screening and might be useful as simple frailty screening tools to identify older adults who might benefit from CGA and integrated, multidisciplinary geriatric care services.

\section{KEY MESSAGE}

1. The Korean Frailty Index (KFI) showed content, construct, and criterion validity when compared to the Cardiovascular Health Study frailty scale in Korean nationwide population of older adults.

2. The modified KFI, which replacing the timed up and go test with a simple mobility question, could similarly predict frailty status as the original KFI.

3. The KFI and modified KFI are valid instruments for frailty screening and might be useful as simple frailty screening tools in Korean older adults. 


\section{Conflict of interest}

No potential conflict of interest relevant to this article was reported.

\section{Acknowledgments}

This research was supported by a grant from the Korea Health Technology R\&D Project through the Korean Health Industry Development Institute (KHIDI), funded by the Ministry of Health and Welfare, Republic of Korea (grant no. HI15C3153).

\section{REFERENCES}

1. Clegg A, Young J, Iliffe S, Rikkert MO, Rockwood K. Frailty in elderly people. Lancet 2013;381:752-762.

2. Jung HW, Jang IY, Lee YS, et al. Prevalence of frailty and aging-related health conditions in older Koreans in rural communities: a cross-sectional analysis of the aging study of Pyeongchang rural area. J Korean Med Sci 2016;31:345-352.

3. Jung HW, Kim SW, Ahn S, et al. Prevalence and outcomes of frailty in Korean elderly population: comparisons of a multidimensional frailty index with two phenotype models. PLoS One 2014;9:e87958.

4. Song X, Mitnitski A, Rockwood K. Prevalence and 10-year outcomes of frailty in older adults in relation to deficit accumulation. J Am Geriatr Soc 2010;58:681-687.

5. Rockwood K, Song X, MacKnight C, et al. A global clinical measure of fitness and frailty in elderly people. CMAJ 2005;173:489-495.

6. Jung HW, Jang IY, Lee CK, et al. Usual gait speed is associated with frailty status, institutionalization, and mortality in community-dwelling rural older adults: a longitudinal analysis of the Aging Study of Pyeongchang Rural Area. Clin Interv Aging 2018;13:1079-1089.

7. Cesari M. Physical frailty and sarcopenia: development of a framework for supporting interventions against incident mobility disability. Ann Geriatr Med Res 2017;21:42-48.

8. Afilalo J, Kim DH. Frailty scales in transcatheter aortic valve replacement: incremental body of evidence. JACC Cardiovasc Interv 2018;11:1537-1538.

9. Ruiz J, Miller AA, Tooze JA, et al. Frailty assessment predicts toxicity during first cycle chemotherapy for advanced lung cancer regardless of chronologic age. J Geriatr Oncol 2019;10:48-54.
10. Kim SW, Han HS, Jung HW, et al. Multidimensional frailty score for the prediction of postoperative mortality risk. JAMA Surg 2014;149:633-640.

11. Dent E, Kowal P, Hoogendijk EO. Frailty measurement in research and clinical practice: a review. Eur J Intern Med 2016;31:3-10.

12. Fried LP, Tangen CM, Walston J, et al. Frailty in older adults: evidence for a phenotype. J Gerontol A Biol Sci Med Sci 2001;56:M146-M156.

13. Searle SD, Mitnitski A, Gahbauer EA, Gill TM, Rockwood K. A standard procedure for creating a frailty index. BMC Geriatr 2008;8:24.

14. Mitnitski AB, Graham JE, Mogilner AJ, Rockwood K. Frailty, fitness and late-life mortality in relation to chronological and biological age. BMC Geriatr 2002;2:1.

15. Goggins WB, Woo J, Sham A, Ho SC. Frailty index as a measure of biological age in a Chinese population. $J$ Gerontol A Biol Sci Med Sci 2005;60:1046-1051.

16. Hwang HS, Kwon IS, Park BJ, Cho B, Yoon JL, Won CW. The validity and reliability of Korean Frailty Index. J Korean Geriatr Soc 2010;14:191-202.

17. Won CW, Lee Y, Choi J, et al. Starting construction of frailty cohort for elderly and intervention study. Ann Geriatr Med Res 2016;20:114-117.

18. Chen LK, Liu LK, Woo J, et al. Sarcopenia in Asia: consensus report of the Asian Working Group for Sarcopenia. J Am Med Dir Assoc 2014;15:95-101.

19. Orme JG, Reis J, Herz EJ. Factorial and discriminant validity of the Center for Epidemiological Studies Depression (CES-D) scale. J Clin Psychol 1986;42:28-33.

20. Jeon SY, Won CW, Choi HR, Kim BS, Kim SY, Hur JH. Physical frailty predicts cognitive decline in elderly people: prospective findings from the living profiles of older people survey in Korea. Korean J Fam Pract 2015;5(Suppl 3):S702-S707.

21. Guralnik JM, Ferrucci L, Pieper CF, et al. Lower extremity function and subsequent disability: consistency across studies, predictive models, and value of gait speed alone compared with the short physical performance battery. J Gerontol A Biol Sci Med Sci 2000;55:M221-M231.

22. Won CW, Yang KY, Rho YG, et al. The development of Korean Activities of Daily Living (K-ADL) and Korean Instrumental Activities of Daily Living (K-IADL) scale. J Korean Geriatr Soc 2002;6:107-120.

23. Kang Y, Na DL, Hahn S. A validity study on the Korean Mini-Mental State Examination (K-MMSE) in dementia 
patients. J Korean Neurol Assoc 1997;15:300-308.

24. Kim KI, Lee JH, Kim CH. Impaired health-related quality of life in elderly women is associated with multimorbidity: results from the Korean National Health and Nutrition Examination Survey. Gend Med 2012;9:309-318.

25. Jung IK, Kwak DI, Joe SH, Lee HS. A study of standardization of Korean Form of Geriatric Depression Scale (KGDS). J Korean Geriatr Psychiatry 1997;1:61-72.

26. Vellas B, Guigoz Y, Garry PJ, et al. The Mini Nutritional Assessment (MNA) and its use in grading the nutritional state of elderly patients. Nutrition 1999;15:116-122.

27. Jung HW, Yoo HJ, Park SY, et al. The Korean version of the FRAIL scale: clinical feasibility and validity of assessing the frailty status of Korean elderly. Korean J Intern
Med 2016;31:594-600.

28. Jang IY, Jung HW, Lee CK, et al. Korean version of the fatigue, resistance, ambulation, illnesses and loss of weight questionnaire versus the modified kihon checklist for frailty screening in community-dwelling older adults: the aging study of PyeongChang rural area. Geriatr Gerontol Int 2017;17:2046-2052.

29. Jeong HS, Lee DW, Park KH, et al. Clinical factors related to frailty estimated by the Korean Frailty Index. J Korean Geriatr Soc 2013;17:71-78.

30. Kim DH, Afilalo J, Shi SM, et al. Evaluation of changes in functional status in the year after aortic valve replacement. JAMA Intern Med 2019;179:383-391. 
Jung HW, et al. Validation of the Korean Frailty Index

Supplementary Table 1. Mean score of KFI, mKFI, and CHS frailty scale in urban and rural dwelling older people in the study

\begin{tabular}{lccccccc}
\hline \multirow{2}{*}{ Score } & \multicolumn{3}{c}{ Men } & & \multicolumn{3}{c}{ Women } \\
\cline { 2 - 3 } & Urban $(\mathrm{n}=683)$ & Rural $(\mathrm{n}=691)$ & pvalue & & Urban $(\mathrm{n}=813)$ & Rural (n=699) & $p$ value \\
\hline KFI & $1.8 \pm 1.5$ & $2.0 \pm 1.6$ & 0.046 & & $2.2 \pm 1.5$ & $2.6 \pm 1.7$ & 0.005 \\
mKFI & $1.6 \pm 1.4$ & $1.7 \pm 1.5$ & 0.024 & & $2.1 \pm 1.6$ & $2.4 \pm 1.7$ & 0.001 \\
CHS frailty scale & $0.6 \pm 0.9$ & $0.8 \pm 1.0$ & $<0.001$ & & $0.9 \pm 0.9$ & $1.3 \pm 1.1$ & $<0.001$ \\
\hline
\end{tabular}

Values are presented as mean \pm SD.

KFI, Korean Frailty Index; mKFI, modified Korean Frailty Index; CHS, Cardiovascular Health Study. 